\title{
SIMULATION OF THE EFFECT OF WIND SPEEDUP IN THE FORMA- TION OF TRANSVERSE DUNE FIELDS
}

\author{
HIROSHI MOMIJI ${ }^{1}$, RICARDO CARRETERO-GONZÁLEZ ${ }^{2} \dagger$, STEVEN R BISHOP $^{2}$ AND ANDREW WARREN ${ }^{1}$ \\ ${ }^{I}$ Department of Geography, University College London, 26 Bedford Way, London WC1H 0AP, UK \\ ${ }^{2}$ Centre for Nonlinear Dynamics and its Applications, University College London, Gower Street, London WC1E 6BT, UK
}

Received 16 September 1999; Revised 21 February 2000; Accepted 25 February 2000

\begin{abstract}
A computer simulation model for transverse-dune-field dynamics, corresponding to a uni-directional wind regime, is developed. In a previous formulation, two distinct problems were found regarding the cross-sectional dune shape, namely the erosion in the lee of dunes and the steepness of the windward slopes. The first problem is solved by introducing no erosion in shadow zones. The second issue is overcome by introducing a wind speedup (shear velocity increase) factor, which can be accounted for by adding a term to the original transport length, which is proportional to the surface height. By incorporating these features we are able to model dunes whose individual shape and collective patterns are similar to those observed in nature. Moreover we show how the introduction of a non-linear shear-velocity-increase term leads to the reduction of dune height, and this may result in an equilibrium dune field configuration. This is thought to be because the non-linear increase of the transport length makes the sand trapping efficiency lower than unity, even for higher dunes, so that the incoming and the outgoing sand flux are in balance. To fully describe the inter-dune morphology more precise dynamics in the lee of the dune must be incorporated. Copyright (C) 2000 John Wiley \& Sons, Ltd.
\end{abstract}

KEY WORDS: simulation; wind speedup; transverse dune; dune field

\section{INTRODUCTION}

Mathematical models are increasingly popular for the understanding of natural phenomena, and are employed to predict future states, possibly including human influences. Here we are interested in the morphology, dune growth, downwind migration and spatial patterns that form in a sand field. In order to develop a useful and accurate model some important factors need to be considered: the wind pattern over a dune must be correctly determined; the induced sand transport must be precisely estimated; and many other physical factors must be incorporated. Some progress has already been made especially with isolated barchan dunes as in the works of Howard et al. (1978), Wippermann and Gross (1985), Zeman and Jensen (1988), Weng et al. (1991) and Van Dijk et al. (1999). Though the question of equilibrium shape was not solved by Wippermann and Gross, they succeeded in reproducing a barchan dune with a good geometrical agreement to natural dunes. However, extending this approach to a dune field, where many dunes grow, migrate and interact, is not an easy task. It is, for example, extremely difficult to calculate three-dimensional wind patterns over many different forms of transient dune, such as partially connected dunes.

Recently, more abstract models to describe dune-field evolution have been developed by Werner (1995) and Nishimori and co-workers (Nishimori and Ouchi, 1993; Nishimori et al., 1998). Though their mathematical formulations are not the same, both approaches assume that a dune field can be considered to be an accumulation of sand 'slabs' piled in a two-dimensional lattice, and that the movement of these slabs can be used to simulate wind directional sand transport and deformational shaping by gravity. Both these models succeeded in reproducing some types of dunes such as barchan dunes, transverse dunes and linear dunes.

\footnotetext{
* Correspondence to: H. Momiji, Department of Geography, University College London, 26 Bedford Way, London WC1H 0AP, UK. E-mail: hmomiji@geog.ucl.ac.uk

$\dagger$ Present address: Department of Mathematics \& Statistics, Simon Fraser University, Burnaby, BC, V5A 1S6, Canada
}

Copyright (C) 2000 John Wiley \& Sons, Ltd. 
They were also able to estimate the relations between dune shapes, wind directional variability, and sand availability, which are roughly confirmed with observations (Wasson and Hyde, 1983; Livingstone and Warren, 1996, figure 5.22, p.80). This modelling approach is thought to become more important for understanding the complex nature of geomorphology, which has not been explained by the conventional 'reductionism' approaches (see for example Werner, 1999). If incorporating the concept of sediment state including sand supply, these models would be able to simulate the evolution of a particular dune field over a long time period (Kocurek and Lancaster, 1999). Werner's simulation results suggest that defects (crest terminations) may be essential for the dune-field dynamics. Werner and Kocurek $(1997,1999)$ have been developing a dune-field model based on the defect dynamics.

Although there have been obvious successes, problems remain in these models. The most acute problem in Nishimori's model is that deformational shaping by gravity and directional sand transport occur at the same time scale, though in nature avalanches occur at faster time scales than bulk sand transport. In the Werner model the cross-sectional views of dunes are much more symmetric than in real dunes, as he noted in his paper. He thought: 'A possible remedy is modifying the transport rules to account for the relationships among local morphology, wind profile, and transport flux in greater detail' (Werner, 1995). Both models can nonetheless create dune-field patterns that approximately resemble natural ones.

We shall restrict our studies to transverse dunes, appearing in infinitely deep sand fields under a unidirectional wind regime. Transverse dunes are thought to be the simplest dunes with regard to shape and configuration. In their paper, Momiji and Warren emphasize wind speedup over a dune and sand trapping efficiency as important factors when discussing equilibrium migration of transverse dunes (Momiji and Warren, 2000).

In this paper, we analyse Werner's dune-field simulation model for the uni-directional wind regime leading to the evolution of transverse dunes. Following this, we introduce two revisions to the Werner model, which lead to dunes that more closely resemble those in nature. The first revision incorporates wind structure over a dune, and the second considers the wind speedup and the equilibrium dune shape.

\section{MODEL AND ALGORITHM}

Though some corrections and revisions are introduced in later sections, the basic model and algorithm used here are predominantly the same as used by Werner (1995). Dunes are built from sand slabs piled either on the one dimensional or on the two-dimensional square lattice, whose edges are connected with periodic boundary conditions. The number of sand slabs is proportional to the surface height, and their movement comprises one-directional transport together with avalanching dynamics.

A sand slab is individually and randomly chosen for transport (erosion) from among all the slabs on the surface. The slab is moved a specific number of lattice sites, thus introducing a transport length $(L)$, and is deposited at a site with a probability $\left(P_{S}\right)$ (following Werner's notation). If the slab is not deposited, then it is repeatedly moved over $L$ sites until deposition. Subsequently another slab is chosen randomly for transport. Shadow zones are introduced in the lee of dunes. If a slab is transported to a site in a shadow zone, it is deposited with unit probability. This process is repeated to construct the time evolution of the dune-field surface.

A maximum difference in surface elevation between adjacent lattice sites is enforced by introducing an angle of repose set to $33 \cdot 7^{\circ}$. If the deposition of a sand slab at a particular site violates the angle-of-repose criterion, the slab is moved down the steepest gradient until compliance is achieved. Similarly, if erosion of a sand slab oversteepens the slope, starting at that lattice site, neighbouring sand slabs are moved downslope one lattice site, successively going up the steepest gradient until no angle exceeds the angle of repose. The only slab movement not in the transport direction originates from this form of avalanching.

The initial morphology is generated by placing sand slabs, one by one, on the lattice at random locations. In Werner's original paper, transverse dunes were simulated from the initial random morphology with 15 slabs per site on average. Here, because they appear in an infinitely deep sand field, 100 slabs are piled on each site on average. Alternatively, dunes are constructed on the level surface with a depth of 100 slabs, as initial conditions. The windward slope angle of the initial dune was set to $10^{\circ}$. In the simulation time denoted by $t$ is 
(a)

(b)
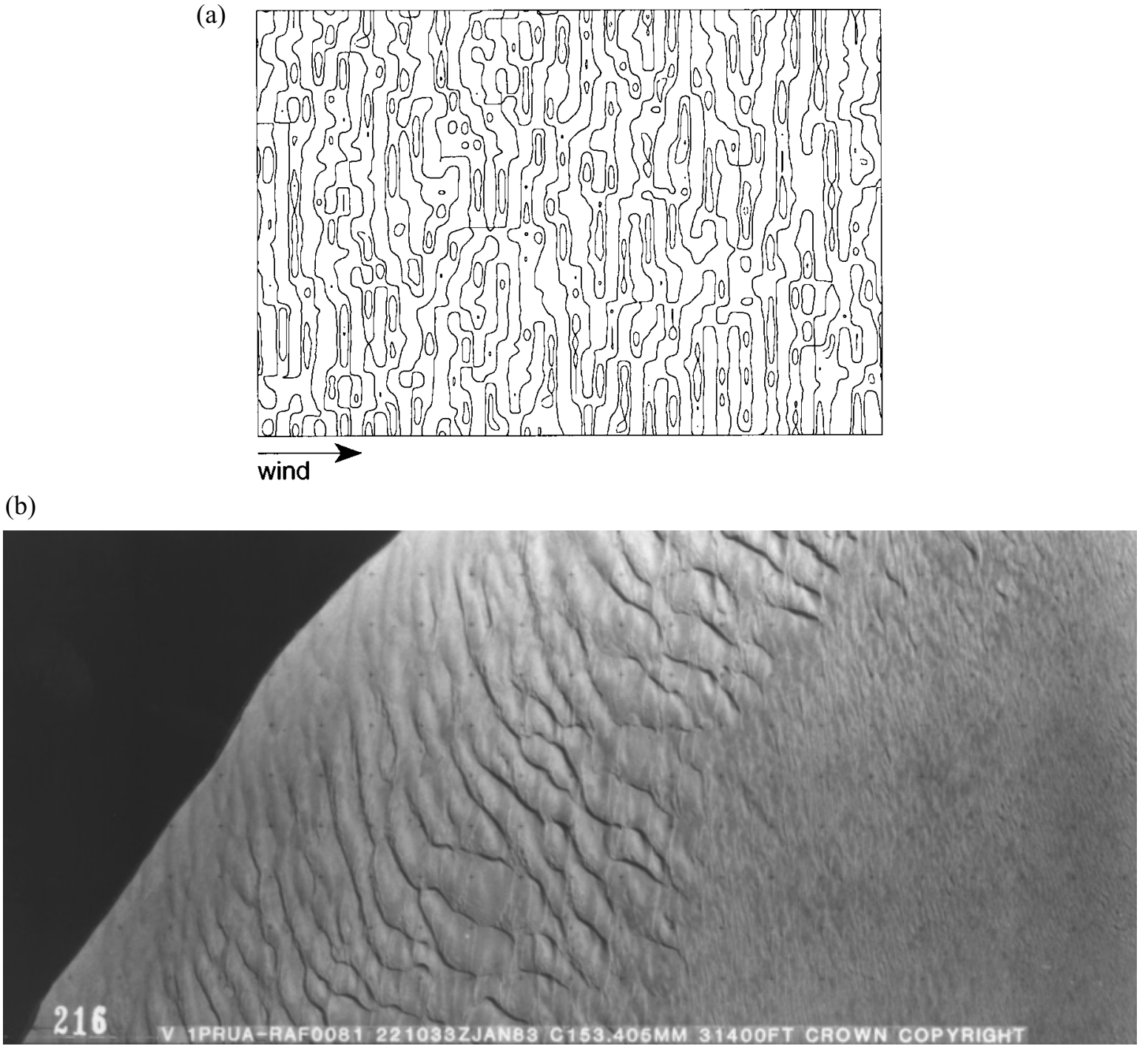

Figure 1. Transverse dunes (a) in $(i, j)$ space simulated with Werner's original model, from a random initial morphology, and (b) in real nature. The simulation time $(t)$ is 500. Contour lines are for 100, 105 and 110 slabs

the number of lattice sites that have been polled for slab erosion divided by the number of surface lattice sites in the simulation. In all simulations, even after $t=1000000$, there were no sites without a sand slab.

The parameters used in the simulations are given in Table I, which are almost the same as those used by Werner.

\section{DUNE SHAPE AND DYNAMICS SIMULATED WITH WERNER'S ORIGINAL MODEL}

Figure 1a shows transverse dunes on a two-dimensional lattice $(1024 \times 1024)$ simulated after $t=500$, from a random initial morphology without any distinctive pattern or orientation, whose minimum and maximum number of slabs are 95 and 104, respectively. The windward slope angles are almost the same as the angle of repose of $33.7^{\circ}$, which is much larger than those of real dunes (about $10^{\circ}$ ). Consequently the resulting dunes have symmetrical cross-sections, though as seen in Figure $1 \mathrm{~b}$ transverse dunes in nature have asymmetrical 
Table I. Simulation parameters

\begin{tabular}{lc}
\hline Parameter & Value \\
\hline Lattice size: & \\
2d lattice & $1024 \times 1024^{*}$ \\
1d lattice* & $1024^{*}$ \\
Slab aspect ratio & $1 / 3$ \\
Angle of repose & $\tan ^{-1}(2 / 3)=33 \cdot 7^{\circ}$ \\
Shadow zone angle & $15^{\circ}$ \\
Transport length & $L=5 \dagger$ \\
Erosion probability & same at every site $\dagger$ \\
Deposition probability: & $P_{s}=0.6$ \\
$\quad$ at a site outside shadow zone & $P_{s}=1.0$ \\
at a site in shadow zones & $100^{*}$ \\
Average number of slabs at a site & \\
\hline
\end{tabular}

* Different from Werner's (1995) simulation

$\uparrow$ Revised in later sections

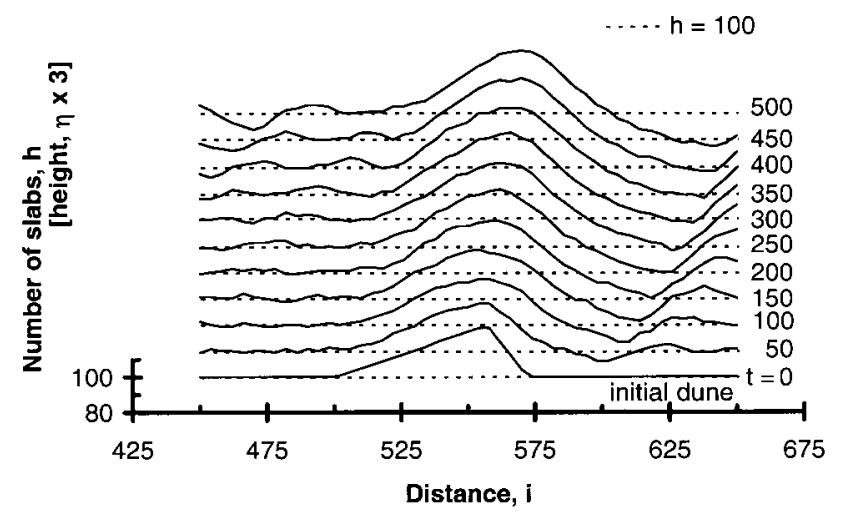

Figure 2. Evolution of an initially introduced, isolated transverse dune, simulated with Werner's original model. The dune's initial number of slabs above the surface, shown by a dotted, line is 30 . The total simulation time $(t)$ is 500

cross-sections with gentler windward slopes. As seen in figure 3A in Werner's (1995) paper, transverse dunes also have symmetrical cross-sections.

Using the same model, Figure 2 shows the cross-sectional views of an evolving isolated transverse dune on a level surface on a two-dimensional lattice, whose initial number of slabs above the surface $(h)$ was 30 . The simulation times $(t)$ are 0 to 500. The dune migrates downwind, during which its windward slope steepens and its slip face in the lee becomes shallower. However, at the same time, the previously level surface upwind and downwind of the dune deform and the windward slope shows undulation. Meanwhile, downwind of the initial dune, smaller accumulations form which themselves grow as time evolves (e.g. close to $i=625$ ). Lower dunes migrate more quickly, but are ephemeral, and as time passes dune patterns of longer wavelength consisting of larger dunes grow and dominate the dune field.

Shorter wavelengths are not sustained long term due to avalanching. Dune envelopes are shaped by sand accumulation and avalanching that suppresses short-wavelength undulations. With regard to avalanching, it can be shown that the wavelength limits the amplitude of undulation, so that only longer-wavelength undulations can grow higher, resulting in dunes with a distinct shape. Assuming that an undulation $(f)$ has a wavelength of $\Lambda$ and a maximum height of $\eta_{\Lambda}$, we may approximate the field as a sinusoidal function:

$$
f=\left(\eta_{\Lambda}-\eta_{\text {avg }}\right)(2 \pi / \Lambda) \sin ((2 \pi / \Lambda) x)
$$


where $x$ is a distance measure and $\eta_{\text {avg }}$ is the average height. The local gradient $(\partial f / \partial x)$ is:

$$
\partial f / \partial x=\left(\eta_{\Lambda}-\eta_{\text {avg }}\right)(2 \pi / \Lambda) \cos ((2 \pi / \Lambda) x)
$$

Since the gradient $(\partial f / \partial x)$ cannot exceed $2 / 3$ which corresponds to the angle of repose of $33 \cdot 7^{\circ}$ then:

$$
\eta_{\Lambda}-\eta_{\text {avg }} \leq(\Lambda / 2 \pi) \tan 33 \cdot 7^{\circ}=(\Lambda / 2 \pi)(2 / 3) \simeq 0 \cdot 11 \Lambda
$$

Consequently, shorter-wavelength undulations can only achieve lower heights, and thus only longerwavelength undulations can grow higher.

Next, it can be shown that sand accumulation is due to the existence of the shadow zones in the lee of dunes, where deposition probability is unity. Without a shadow zone, the sand slab (s. s.) conservation equation can be described as:

$$
\begin{gathered}
h_{n+1}(i, j)=h_{n}(i, j)+(\text { transported-incoming s. s. }) \\
-(\text { transported-outgoing (erosion) s.s })+\text { avalanche } \\
=h_{n}(i, j)+\left(\sum_{i^{\prime}=i-k L}\left(1-P_{s}\right)^{k-1} P_{s}+\text { by-passing s.s }\right) \\
-(1+\text { by-passing s.s })+\text { avalanche }
\end{gathered}
$$

where $h_{n}(i, j)$ is the number of slabs at the site $(i, j)$ at time $n$. Given that the system is infinitely large, the second term of the right-hand side of Equation 1 is:

$$
\sum_{i^{\prime}=i-k L}\left(1-P_{s}\right)^{k-1} P_{S} \rightarrow P_{S} \sum_{k}^{\infty}\left(1-P_{s}\right)^{k-1}=P_{s} /\left\{1-\left(1-P_{s}\right)\right\}=1
$$

Because avalanching occurs equally at each site, Equation 1 implies that on average, taken over all sites with a periodic boundary condition:

$$
h_{n+1}(i, j)-h_{n}(i, j)=0
$$

In the case of infinite sand depth, producing transverse dunes in Werner's model, dunes grow because incoming sand is accumulated once it has been deposited in the shadow zone, either directly or via avalanching. Without a shadow zone, from Equations 1 and 2 the sand flux is found to be constant at every site.

\section{NO EROSION IN SHADOW ZONES}

In Werner's original model the surface in the lee of a dune is eroded. In his model, shadow zones are applied only to sand slab deposition, and erosion still occurs in the shadow zones. A recent field study shows that the wind flow pattern in the lee of the dune is very complex, and that there is apparent sand transport even in the separation cell, which corresponds to the shadow zone in the model (Walker, 1999). However, it is thought to be a good first approximation that the wind speed within the separation cell is typically insufficient to cause sand movement (Frank and Kocurek, 1996a). Figure 3 shows cross-sectional views of transverse dunes simulated without erosion in shadow zones, on a two-dimensional lattice from an initially isolated transverse 


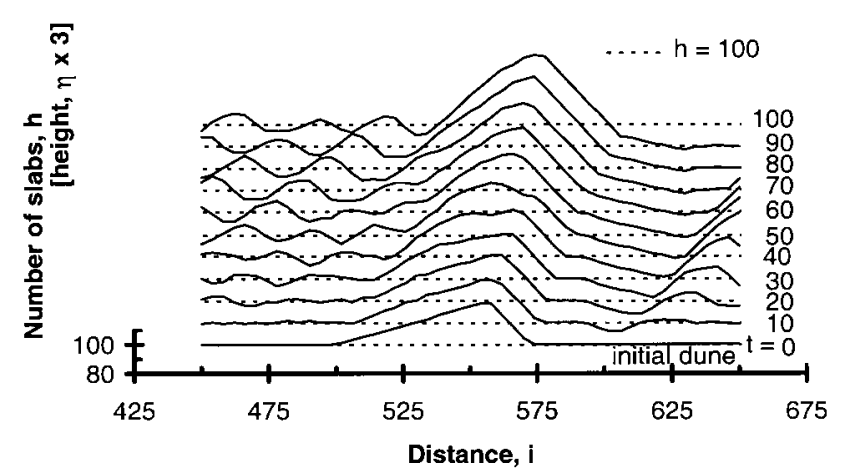

Figure 3. Evolution of an initially introduced, isolated transverse dune simulated without erosion in shadow zones. The dune's initial number of slabs above the surface is 30 . The simulation time $(t)$ is 100

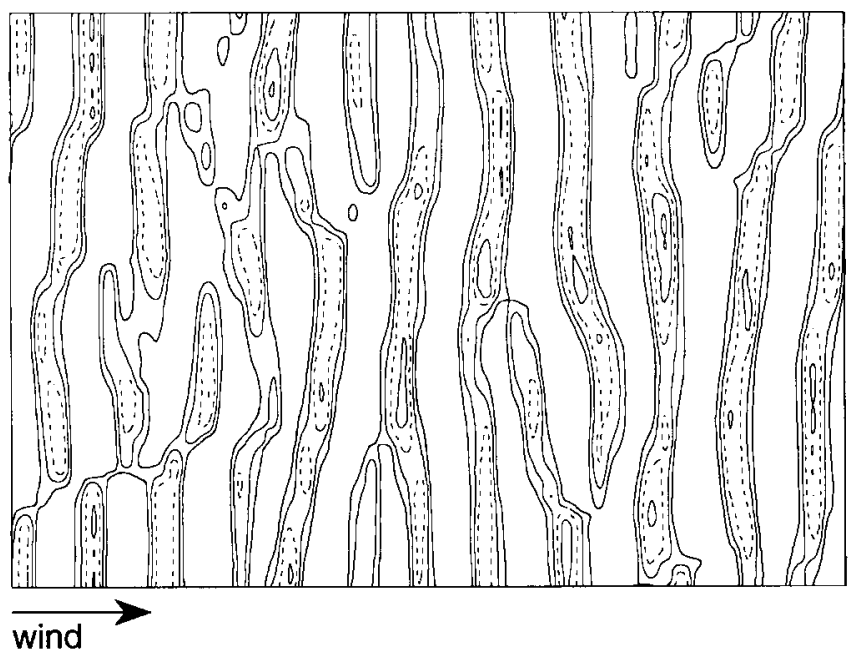

Figure 4. Transverse dunes simulated from an initially random morphology. The simulation time $(t)$ is 500 . No erosion occurs in shadow zones. Contour lines are for 100,110,120, 130 and 140 slabs

dune with number of slabs $(h)$ of 30 , built on a level surface. The simulation times $(t)$ are 0 to 100 . With no erosion in the shadow zones: (a) the slip face in the lee is sustained; (b) the dunes grow more quickly due to strong accumulation of sand slabs; and (c) the dunes migrate downwind with constant speed. These features are similar to those of dunes in nature. Figure 4 shows transverse dunes simulated on a two-dimensional lattice from an initially random morphology. Without erosion in the shadow zones, the dunes are higher than those shown in Figure 1 owing to strong slab accumulation. In the remainder of this paper we consider no erosion in shadow zones in all simulations.

\section{INTRODUCING WIND SPEEDUP}

The wind speedup over a windward slope of a dune is necessary to sustain the shape of the windward slope (Bagnold, 1941; Zeman and Jensen, 1988; (Momiji and Warren, 2000). This wind speedup has been noted, both in the field and in the wind tunnel experiments (see for example Lancaster et al., 1996; Mulligan, 1988; Wiggs et al., 1996; Frank and Kocurek, 1996b). Mulligan found that the wind speed increases linearly over a transverse dune. The more important quantity here is wind shear velocity $(u *)$, or equivalently the wind shear 
stress, that causes sand transport. Lancaster and co-workers found that the shear velocity increases linearly over a barchan dune, provided that the wind is strong enough such that the shear velocity significantly exceeds the threshold shear velocity (Lancaster et al., 1996, figure 10).

In a revised model, Nishimori and co-workers attempted to introduce a more realistic slab transport rule compared to Werner's approach, based on the one-dimensional wind flow calculation (two-dimensional wind flow pattern) over a well-developed dune, in which wind speedup is included (Nishimori et al., 1998).

In this paper, we attempt to introduce a wind speedup effect into Werner's model with a kinematic rule. Following this, we examine the effect of wind speedup on a dune shape with some simulations.

\section{Kinematic formulation}

If dunes migrate downwind at the constant speed $\left(c_{d}\right)$ without changing their shapes as is widely believed (Bagnold, 1941), then the local height $(\eta(x, t))$ follows the advection equation:

$$
\partial \eta / \partial t+c_{d} \partial \eta / \partial x=0
$$

Combining Equation 3 and the sand conservation equation:

$$
\gamma \partial \eta / \partial t+\partial q / \partial x=0
$$

where $\lambda$ is the sand bulk density in dunes and $q$ is sand flux, we obtain:

$$
\gamma c_{d} \partial \eta / \partial x=\partial q / \partial x
$$

Equation 4 must always be satisfied when we discuss the migration of shape-invariant dunes (Bagnold, 1941; Zeman and Jensen, 1988). Integrating Equation 4 from $x=-\infty$ to $x<0$ corresponding to the dune crest leads to:

$$
q(x)-q(-\infty)=\gamma c_{d} \eta(x)
$$

Let us assume that sand flux $(q(x))$ obeys Bagnold's sand transport formula:

$$
q(x)=C(d / D)^{1 / 2}\left(\rho_{a} / g\right)\left(u_{*}(x)\right)^{3}
$$

where $C$ is a constant, $d$ is sand grain diameter, $D$ is reference diameter of $0.25 \mathrm{~mm}, \rho_{a}$ is air density and $g$ is gravitational acceleration. Assuming that the shear velocity on a dune surface is $\delta u_{*}(x)$ larger than that on bare ground, due to the wind speedup over the dune, the corresponding increase of sand flux is written as:

$$
\begin{gathered}
q(x)-q(-\infty)=C(d / D)^{1 / 2}\left(\rho_{a} / g\right)\left[u_{*}(x)^{3}-u_{*}(-\infty)^{3}\right] \\
=C(d / D)^{1 / 2}\left(\rho_{a} / g\right)\left[\left(u_{*}(-\infty)+\delta u_{*}(x)\right)^{3}-u_{*}(-\infty)^{3}\right] \\
=C(d / D)^{1 / 2}\left(\rho_{a} / g\right) u_{*}(-\infty)^{3}\left[\left\{1+\left(\delta u_{*}(x) / u_{*}(-\infty)\right)\right\}^{3}-1\right]
\end{gathered}
$$

If the increase of shear velocity is small enough:

$$
\delta u_{*}(x) \ll u_{*}(-\infty)
$$


Equation 6 becomes:

$$
\begin{gathered}
q(x)-q(-\infty)=C(d / D)^{1 / 2}\left(\rho_{a} / g\right) u_{*}(-\infty)^{3}\left[\left\{1+3\left(\delta u_{*}(x) / u_{*}(-\infty)\right)\right\}-1\right] \\
\approx 3 C(d / D)^{1 / 2}\left(\rho_{a} / g\right) u_{*}(-\infty)^{2} \delta u_{*}(x)
\end{gathered}
$$

Combining Equations 5 and 7:

$$
\delta u_{*}(x) \approx\left[\left(\gamma c_{d}\right) /\left\{3 C(d / D)^{1 / 2}\left(\rho_{a} / g\right) u_{*}(-\infty)^{2}\right\}\right] \eta(x)
$$

Though in Werner's model, both the transport length of sand slabs $(L)$ and the number of slabs to be removed $(\delta h)$ are constant (typically $L=5$ and $\delta h=1), L$ is assumed here to be proportional to $u *(x)$, so that from Equation 8:

$$
L(x)=L_{0}+\delta L_{c} h(x)
$$

where $h(x)$ is the number of slabs at $x$. Given that the sand flux $(q(x))$ can be approximated as:

$$
q(x)=L(x) \delta h(x) / 3
$$

where the coefficient 3 is the inverse slab aspect ratio (Nishimori et al., 1998), since

$$
q(-\infty)=L_{0} \delta h(x) / 3 \approx u_{*}(-\infty)^{3}
$$

and

$$
\delta q(x)=\delta L(x) \delta h(x) / 3 \approx u_{*}(-\infty)^{2} \delta u_{*}(x)
$$

$\delta h(x)$ must be given by:

$$
\delta h(x) \approx u_{*}(-\infty)^{2}=\text { const } .
$$

Consequently from Equations 9 and 10, the wind speedup over a dune can be introduced by letting the transport length of sand slab $(L)$ be:

$$
L(x)=L_{0}+\delta L_{c}\left(h(x)-h_{\text {avg }}\right)
$$

where $x$ is the erosion/ongoing 'bounce' site and $h_{\text {avg }}$ is the average number of slabs, here taken as 100 . Equation 11 can be interpreted as the linear shear velocity increase observed by Lancaster et al. (1996).

\section{Simulations}

Simulations were carried out with and without a wind speedup term introduced in the form of Equation 11. Figure 5 shows the simulation results started from the same initial transverse dune used above, with various shear-velocity-increase coefficients $\left(\delta L_{c}\right)$. Introducing wind speedup makes: (a) dunes lower; (b) dunes migrate more quickly; and (c) dune windward slopes gentler, producing asymmetric dunes. For $\delta L_{c}=0 \cdot 4$, windward slopes with the angle of $10^{\circ}$ are sustained. The dune did not vanish even with a higher shearvelocity-increase coefficient, say $\delta L_{c}=0 \cdot 6$, after $t=1000000$. Figure 6 shows asymmetric transverse dunes formed on a two-dimensional lattice from a random initial morphology by introducing wind speedup. The 


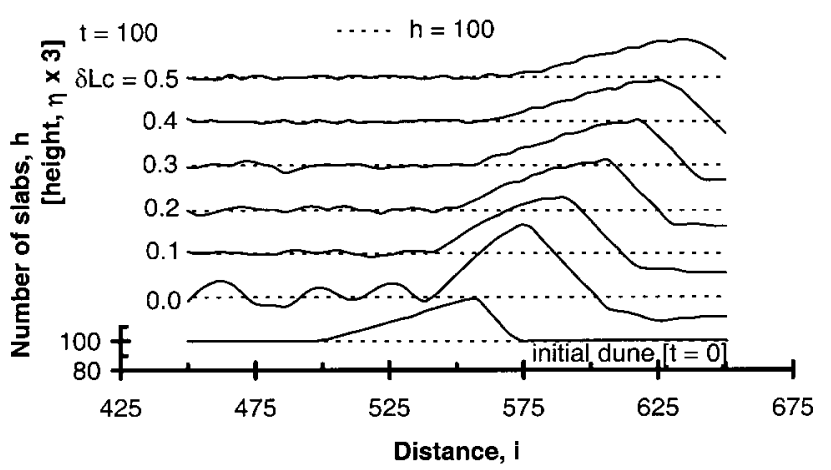

Figure 5. Evolution of an initially introduced, isolated transverse dune simulated with various shear-velocity-increase coefficients $\left(\delta L_{c}\right)$. The dune's initial number of slabs above the surface is 30 . The simulation time $(t)$ is 100

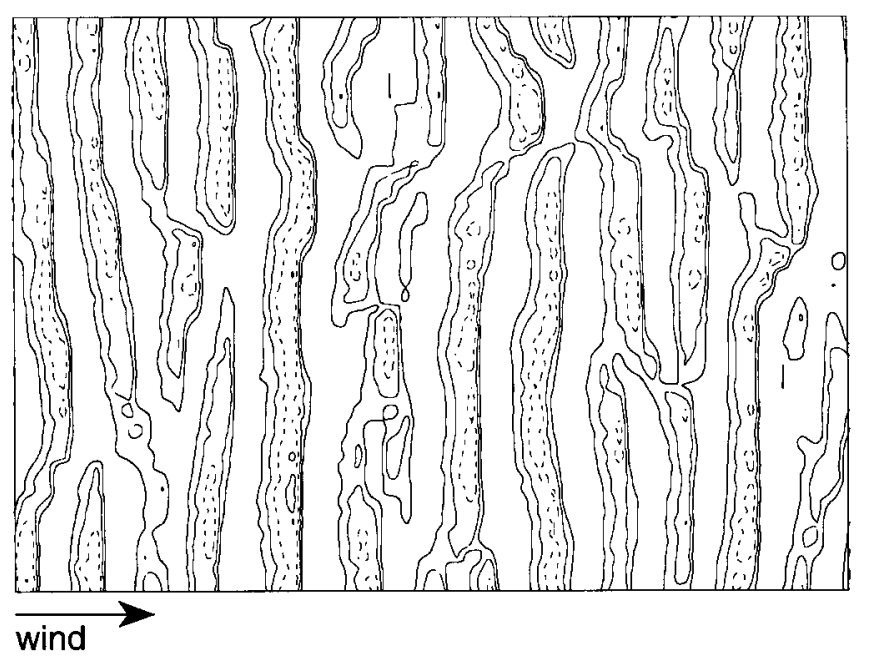

Figure 6. Asymmetric transverse dunes formed from a random initial morphology by introducing wind speedup. The simulation time $(t)$ is 1500 , and the shear-velocity-increase coefficient $\left(\delta L_{c}\right)$ is $0 \cdot 4$. Contour lines are for $100,105,110$ and 115 slabs

simulation time $(t)$ is 1500 , and the shear-velocity-increase coefficient $\left(\delta L_{c}\right)$ is $0 \cdot 4$. The resulting transverse dunes are the most qualitatively similar to natural ones achieved yet.

The time evolution of a one-dimensional dune field with the shear-velocity-increase coefficient $\left(\delta L_{c}\right)$ of $0 \cdot 4$ is shown in Figure 7. The wavelength of the transverse dunes increases as time passes. For run times longer than that displayed in the figure, the equilibrium dune field seems to converge to only an isolated, asymmetric dune, which is surrounded by completely plane, bare ground. Consequently, the dune-to-dune spacing is equal to the lattice size, 1024, meaning that the model is not adequate when the dune-to-dune spacing approaches the lattice size.

The reason for this equilibrium dune-field configuration is thought to be related to sand trapping efficiency $\left(T_{E}\right)$, which is the proportion of moving sand trapped in the dune slip face in the lee (Wilson, 1972; Cooke et al., 1993). At equilibrium, sand flux coming to the dune must be the same as the outgoing flux (Zeman and Jensen, 1988; (Momiji and Warren, 2000). However, because the shadow zone extends more quickly than the slab transport length at the crest of dune $(L)$ increases as the dune grows higher, all the slabs crossing the dune crest will be captured in the dune, meaning the sand trapping efficiency $\left(T_{E}\right)$ is equal to $1 \cdot 0$, so that nothing 


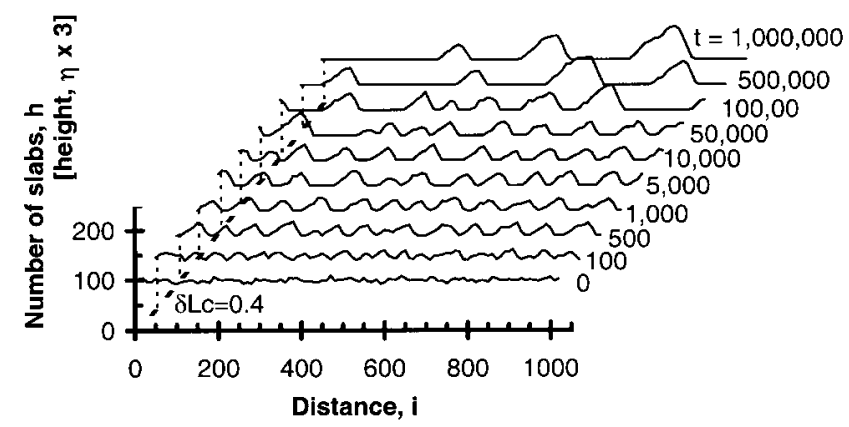

Figure 7. Time evolution of a one-dimensional dune field, from a random initial morphology. The shear-velocity-increase coefficient $\left(\delta L_{c}\right)$ is $0 \cdot 4$.

regulates dune height. Wind speedup effect introduced with the form of Equation 11, seems to regulate only the windward slope angle.

\section{NON-LINEAR WIND SPEEDUP EFFECTS AND EQUILIBRIUM DUNE FIELD}

In the previous section, by assuming that the increase of shear velocity is small enough $(\delta u *(x)<<u *(-\infty))$, the linear slab transport was discussed. In the field, however, this condition is not always satisfied. The nonlinear increase of shear velocity was observed by Frank and Kocurek (1996b, figure 3). More importantly, saltation length, which corresponds to the slab transport length $(L)$ in our model, non-linearly increases as shear velocity $\left(u_{*}\right)$ increases. From their microscale analysis of saltating sand grains, Anderson and Hallet established a set of equations describing saltation length $(\lambda)$ against shear velocity $(u *)$ (Anderson and Hallet, 1986; Anderson, 1988). Figure 8 shows the relation between the saltation length $(\lambda)$ and the shear velocity $(u *)$, calculated using equations 2 to $5 \mathrm{~b}$ in Anderson (1988). Other parameters used here are sand grain diameter of $0.25 \mathrm{~mm}$ and lift-off angle of $50^{\circ}$ (White and Schulz, 1977). Considering these two non-linear relations about shear velocity $\left(u_{*}\right)$, it seems to be reasonable that the non-linear shear-velocity-increase term $\left(\delta L_{c 2}\right)$ is introduced into slab transport length $(L)$ in our model, so that Equation 11 can be revised as:

$$
\begin{array}{rlrl}
L(x) & =L_{0}+\delta L_{c}\left(h(x)-h_{\text {avg }}\right)+\delta L_{c 2}\left(h(x)-h_{\text {avg }}\right)^{2} & & \left(h(x) \geq h_{\text {avg }}\right) \\
& =L_{0}+\delta L_{c}\left(h(x)-h_{\text {avg }}\right) & \left(h(x)<h_{\text {avg }}\right)
\end{array}
$$

Figure 9 shows the time evolution of a one-dimensional dune field with non-linear increase of the transport length introduced in the form of Equation 12. The shear-velocity-increase coefficient $\left(\delta L_{c}\right)$ is 0.4 and the nonlinear shear-velocity-increase coefficient $\left(\delta L_{c 2}\right)$ is taken as 0.002 . Comparing Figures 7 and 9 , we find that introducing non-linear increase of the transport length (a) makes dunes lower and (b) increases the number of dunes.

Momiji and Warren examined published field data, and found that dune migration speed $\left(c_{d}\right)$ could be approximated as:

$$
c_{d} \simeq a_{c_{d}}+b_{c_{d}}(1 / H)
$$

where $a_{c_{d}}$ and $b_{c_{d}}$ are positive constants and $H$ is dune height. They showed that Equation 13 could theoretically be derived by assuming that the dunes were in equilibrium (Momiji and Warren, 2000). If the 


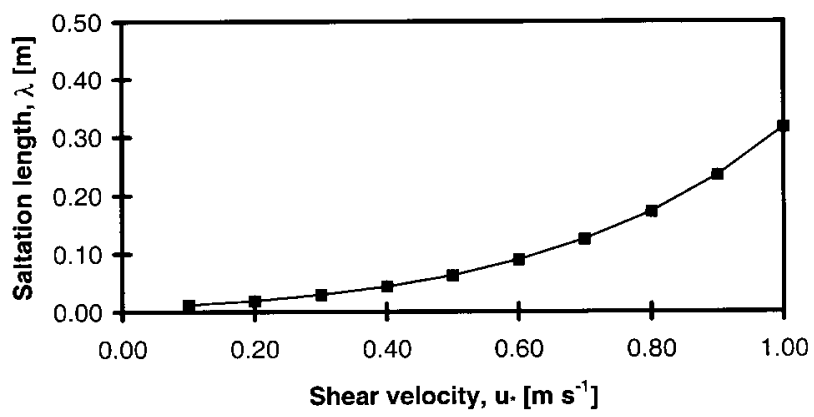

Figure 8. Relation between saltation length $(\lambda)$ and shear velocity $\left(u_{*}\right)$. Data are plotted following Anderson (1988). Other parameters, not specified in his paper but used here, are sand grain diameter of $0.25 \mathrm{~mm}$ and lift-off angle of $50^{\circ}$ (White and Schulz, 1977)

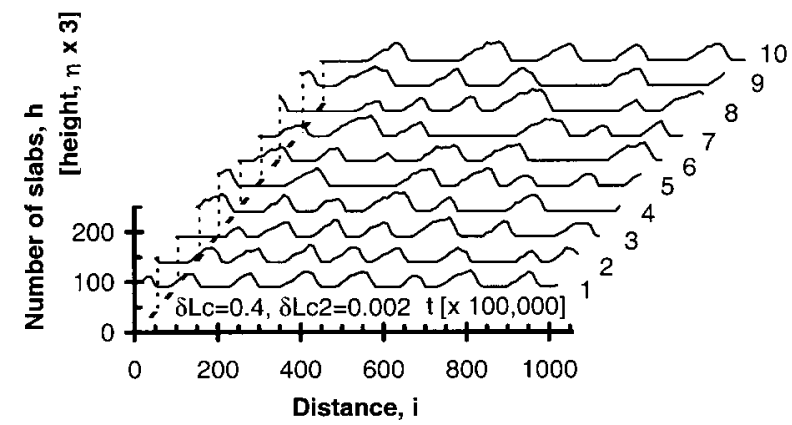

Figure 9. Time evolution of transverse dunes simulated on the one-dimensional lattice with non-linear increase of the transport length, from a random initial morphology. Linear shear-velocity-increase coefficient $\left(\delta L_{c}\right)$ is $0 \cdot 4$. Nonlinear shear-velocity-increase coefficient $\left(\delta L_{c 2}\right)$ is 0.002

dune is nearly at equilibrium but slowly growing, by considering the dimensionality, this near-equilibrium dune can be characterized as:

$$
\partial H / \partial t \ll c_{d}
$$

Combining Equations 13 and 14 leads to:

$$
\partial H(t) / \partial t \ll b_{c_{d}} / H(t)<a_{c_{d}}+b_{c_{d}}(1 / H(t))
$$

Integrating Equation 15 we obtain:

$$
H(t) \ll t^{1 / 2}
$$

Figure 10 shows the relations between maximum dune height and simulation time $(t)$ in the cases with and without non-linear increase of the transport length. $\delta L_{c}$ and $\delta L_{c 2}$ are the same as in Figure 9. In the case with non-linear increase of the transport length, the maximum dune height increases more slowly. The data are well correlated to the square root of time $\left(t^{1 / 2}\right)$, suggesting that the dune field is in near-equilibrium, and even at $t=1000000$, the exact equilibrium state is not reached. The dune height far after $t=1000000$ is thought to be capped by the non-linear wind speedup effect, because some part of slab flux crossing at the crest of a dune goes outside the shadow zone, reducing sand trapping efficiency $\left(T_{E}\right)$ below $1 \cdot 0$, so that the incoming and 


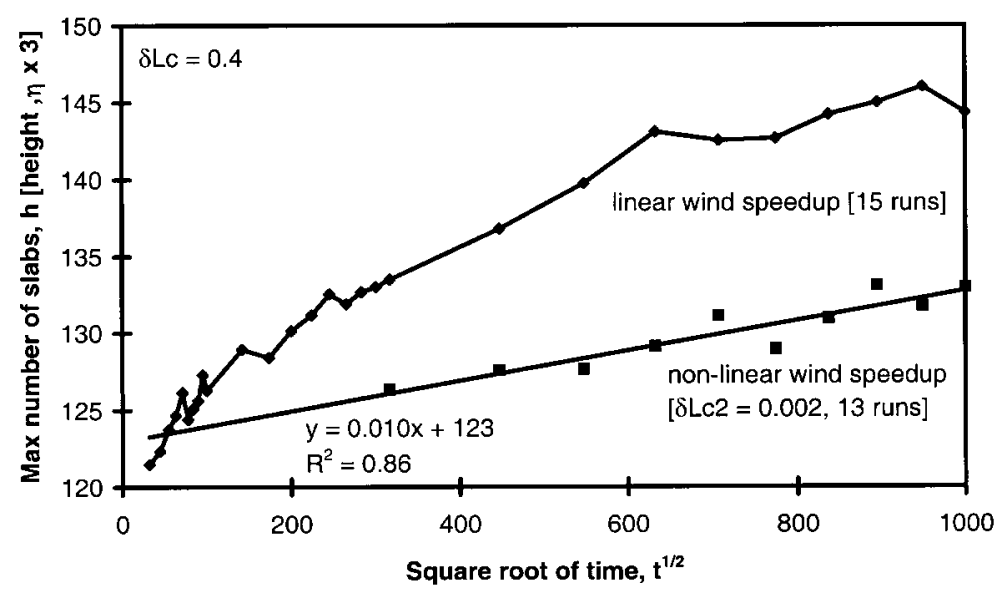

Figure 10. Relations between maximum dune height and simulation time $(t)$. The relations with and without the non-linear increase of the transport length are averaged from 13 and 15 runs on the one-dimensional lattice, respectively. Linear shear-velocity-increase coefficient $\left(\delta L_{c}\right)$ is $0 \cdot 4$. Non-linear shear-velocity-increase coefficient $\left(\delta L_{c 2}\right)$ is 0.002

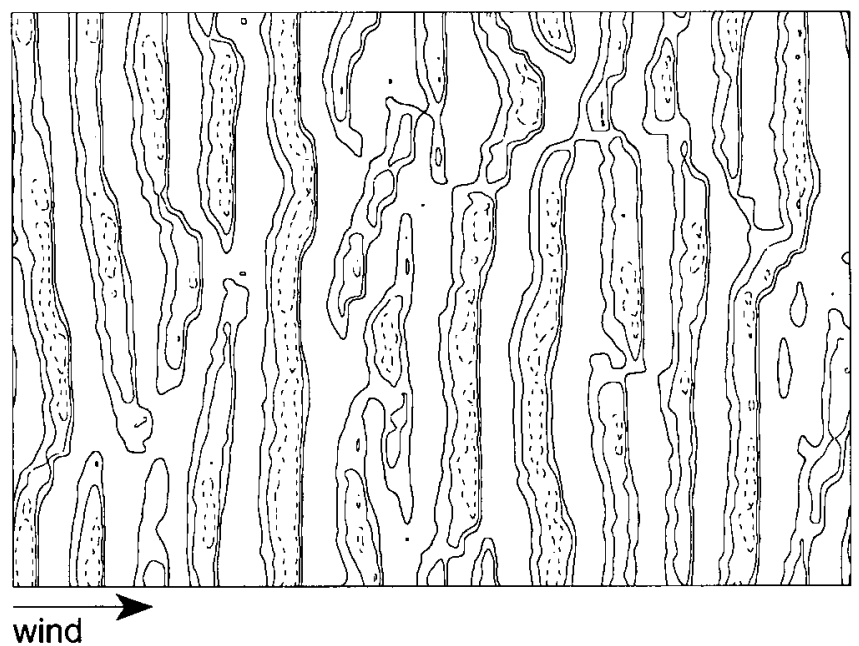

Figure 11. Transverse dunes simulated on the two-dimensional lattice, from a random initial morphology. Linear shear-velocity-increase coefficient $\left(\delta L_{c 2}\right)$ is $0 \cdot 4$. Non-linear shear-velocity-increase coefficient $\left(\delta L_{c 2}\right)$ is $0 \cdot 002$. The simulation time $(t)$ is 1500 . Contour lines are for 100,105 and 110 slabs

outgoing slab fluxes balance. Lancaster et al. reported that shear velocity reached $0.6 \mathrm{~m} \mathrm{~s}^{-1}$ at the site on the windward surface, whose horizontal distance is $35 \mathrm{~m}$ from the toe of the dune (Lancaster et al., 1996, figure 10). The height of this dune was $5 \mathrm{~m}$. According to Momiji and Warren, 2000), the leakage sand flux is very likely to occur for this combination of shear velocity and dune height. To check if the dune height saturates or not, more simulations are needed with longer time $(t)$. Figure 11 shows transverse dunes simulated on the two-dimensional lattice, from a random initial morphology. $\delta L_{c}$ and $\delta L_{c 2}$ are 0.4 and 0.002 , respectively, as in Figures 9 and 10. Simulation time $(t)$ is 1500 . Some dunes in Figure 11 are lower than corresponding dunes in Figure 6 because of the non-linear increase of the transport length.

As seen in Figures 7 and 9, after long enough time, flat surfaces appear between dunes. Following Zeman and Jensen, by considering the relationship between the sand flux $(q(x))$ and the surface height $(\eta(x))$ around the dune in equilibrium, we know that these inter-dune flat surfaces are related to the shadow zones in the lee of dunes, where no slab movement occurs (Zeman and Jensen, 1988, equation 10). However, some transverse 
dune systems observed in nature do not have distinct inter-dune flat surfaces. A field study shows there is complex but apparent sand transport even in the separation cell (Walker, 1999). To describe those features correctly, more precise dynamics in the lee of the dune must be incorporated.

Considering erosion in the shadow zone, the non-linear shear-velocity-increase coefficient $\left(\delta L_{c 2}\right)$ may be over-estimated. In Figure 9, dune heights after a long time period differ significantly in the cases with and without non-linear increase of the transport length. Since the linear slab transport formula (Equation 11) should be the approximation of the non-linear slab transport formula (Equation 12), linear shear-velocityincrease coefficient $\left(\delta L_{c}\right)$ of 0.4 and non-linear shear-velocity-increase coefficient $\left(\delta L_{c 2}\right)$ of 0.002 may not be a good choice of combination. The good parameters combination will be searched with a better formulation of lee-side dynamics.

\section{CONCLUSIONS}

A simulation model for a transverse dune field, corresponding to the uni-directional wind regime, is developed based on Werner's model (1995). In the present work, two distinct problems were overcome. Inconsistencies with the cross-sectional dune shape can be solved by introducing no erosion in shadow zones. The steepness of the windward slope can be correctly modelled by introducing wind speedup over a dune, which can be formulated by adding the height-proportional increase term to the original transport length $(L)$. Introducing a non-linear shear-velocity-increase term can reduce the dune height, which may result in an equilibrium dune-field configuration. This is thought to be because the non-linear increase of the transport length makes the sand trapping efficiency $\left(T_{E}\right)$ lower than 1.0, even for high dunes, so that the incoming and the outgoing sand fluxes balance. To fully describe the inter-dune morphology, more precise dynamics in the lee of the dune must be incorporated.

\section{REFERENCES}

Anderson RS. 1988. The pattern of grainfall deposition in the lee of aeolian dunes. Sedimentology 35: 175-188.

Anderson RS, Hallet B. 1986. Sediment transport by wind: toward a general model. Geological Society of America Bulletin 97: 523535.

Bagnold RA. 1941. The Physics of Blown Sand and Desert Dunes. Methuen; London.

Cooke RU, Warren A, Goudie AS. 1993. Desert Geomorphology. University College Press; London.

Frank A, Kocurek G. 1996a. Toward a model for airflow on the lee side of aeolian dunes. Sedimentology 43: 451-458.

Frank AJ, Kocurek G. 1996b. Airflow up the stoss slope of sand dunes: limitations of current understanding. Geomorphology 17: 4754.

Howard AD, Morton JB, Gad-el-Hak M, Pierce, DB. 1978. Sand transport model of barchan dune equilibrium. Sedimentology 25: 307338.

Kocurek G, Lancaster N. 1999. Aeolian system sediment state; theory and Mojave Desert Kelso dune field example. Sedimentology 46: $505-515$.

Lancaster N, Nickling WG, McKenna Neuman CK, Wyatt VE. 1996. Sediment flux and airflow on the stoss slope of a barchan dune. Geomorphology 17: 55-62.

Livingstone I, Warren A. 1996. Aeolian Geomorphology. Addison Wesley Longman; Harlow, UK.

Momiji H, Warren A. 2000. Relations of sand trapping efficiency and migration speed of transverse dunes to wind velocity. Earth Surface Processes and Landforms in press.

Mulligan KR. 1988. Velocity profiles measured on the windward slope of a transverse dune. Earth Surface Processes and Landforms 13: $573-582$.

Nishimori H, Ouchi N. 1993. Formation of ripple patterns and dunes by wind-blown sand. Physical Review Letters 71: 197-200.

Nishimori H, Yamasaki M, Andersen KH. 1998. A simple model for the various pattern dynamics of dunes. International Journal of Modern Physics B 12: 257-272.

Van Dijk PM, Arens SM, Van Boxel JH. 1999. Aeolian processes across transverse dunes. II: modelling the sediment transport and profile development. Earth Surface Processes and Landforms 24: 319-333.

Walker IJ. 1999. Secondary airflow and sediment transport in the lee of a reversing dune. Earth Surface Processes and Landforms 24: 437-448.

Wasson RJ, Hyde R. 1983. Factors determining desert dune type. Nature 304: 337-339.

Weng WS, Hunt JCR, Carruthers DJ, Warren A, Wiggs GFS, Livingstone I, Castro I. 1991. Air flow and sand transport over sand dunes. Acta Mechanica Supplement 2:1-22.

Werner BT. 1995. Eolian dunes: computer simulations and attractor interpretation. Geology 23: 1107-1110.

Werner BT. 1999. Complexity in natural landform patterns. Science 284: 102-104.

Werner BT, Kocurek G. 1997. Bed-form dynamics: Does the tail wag the dog? Geology 25: 771-774.

Werner BT, Kocurek G. 1999. Bedform spacing from defect dynamics. Geology 27: 727-730. 
White BR, Schulz JC. 1977. Magnus effect in saltation. Journal of Fluid Mechanics 81: 497-512.

Wiggs GFS, Livingstone I, Warren A. 1996. The role of streamline curvature in sand dune dynamics: evidence from field and wind tunnel measurements. Geomorphology 17: 29-46.

Wilson IG. 1972. Aeolian bedforms- their development and origins. Sedimentology 19: 173-210.

Wipperman FK, Gross G. 1985. The wind-induced shaping and migration of an isolated dune: a numerical experiment. BoundaryLayer Meterology, 36: 319-334.

Zeman O, Jensen NO. 1988. Progress report on modeling permanent form sand dunes. RisØ National Laboratory Report RISØ-M2738, Roskilde, Denmark. 